\title{
DIGITALTECHNOLOGY A new reality: Bedside geriatric teaching in an age of remote learning
}

\author{
Authors: Damir Rafi, ${ }^{A *}$ Ashlyn A Stackhouse, ${ }^{A *}$ Risheka Walls, ${ }^{B}$ Melanie Dani, ${ }^{C}$ Adrian Cowell, ${ }^{D}$ Elizabeth Hughes ${ }^{\mathrm{E}}$ \\ and Amir $\mathrm{H} \mathrm{Sam}^{\mathrm{F}}$
}

\begin{abstract}
Population ageing poses many challenges in the landscape of general medicine. One of these is the requirement for more geriatricians to minimise future strain on healthcare systems. Previous studies have identified that, while demand for geriatricians is on the rise, interest among medical students for a career in geriatrics falls behind. We utilised a new augmented reality technology (the Microsoft HoloLens 2) to deliver students a remote bedside teaching experience. Features of this teaching included bi-directional communication, exposure to physical signs, and use of holograms around the patient bedside as a teaching aid. Given its convenience, scope and engagement potential, this educational tool has potential to be used effectively throughout medical training.
\end{abstract}

KEYWORDS: education, remote learning, mixed-reality technology, geriatrics

DOI: $10.7861 /$ fhj.2021-0108

\section{Introduction}

It is projected that over the coming 3 decades in the UK, there will be a shift in the age structure of the population, with an overall higher life expectancy and larger proportions of the population in each of the age brackets over 60 years. Most substantially, the proportion of people aged over 85 years will almost double over the next 25 years. ${ }^{1}$ Geriatrics as a specialty, like many others within the medical profession, suffers from being under-doctored and overstretched, with a need for more higher trainees. If not addressed, this need will only become more prominent given the current UK population projections.

Authors: ${ }^{\text {A }}$ clinical education fellow, Imperial College School of Medicine, London, UK; ${ }^{B}$ digital development lead, Imperial College School of Medicine, London, UK and Imperial College Healthcare NHS Trust, London, UK; ${ }^{C}$ consultant in general and geriatric medicine, Imperial College Healthcare NHS Trust, London, UK; ${ }^{D}$ new media and digital innovation lead, Imperial College School of Medicine, London, UK; ${ }^{E}$ deputy medical director, Health Education England, London, UK; F head, Imperial College School of Medicine, London, UK; *joint first authors
Previous studies have attempted to ascertain medical student perceptions of geriatrics as a specialty to potentially aid recruitment strategies. ${ }^{2}$ In a UK study, while a majority of students considered geriatrics to be intellectually stimulating, over half associated it with low prestige within the medical profession and with a lack of research opportunities. ${ }^{3}$ An Irish-based study exploring similar themes found that medical students commonly considered geriatrics to be an interesting field, with clinical variety common. However, deterrents including a perceived slowness-of-pace. ${ }^{4}$ Here, we demonstrate a new educational tool (augmented reality (AR)) and how it has been used for the first time, to the best of our knowledge, in geriatrics as part of the undergraduate teaching curriculum. We then discuss how innovations such as these can be used to demonstrate the dynamic nature of the profession.

\section{Methods}

\section{Technology}

AR is a form of technology that superimposes digital interfaces upon the physical world. As a result of recent advances in visual technology, the use of AR in medical education has increased over recent years. The fields of anatomy, clinical skills training and surgical simulation have started to integrate AR into their training programmes.

One pioneering AR device is Microsoft HoloLens 2. This has been shown to be a practical, acceptable and effective means to deliver remote-access interactive clinical teaching. ${ }^{5}$ It consists of a headset with a built-in camera to show the wearer's field of vision. The environment, images and holograms that the wearer, namely the tutor, sees can be live-streamed to students' devices, allowing individuals to be able to remotely 'see' through the tutor's eyes. HoloLens 2 further allows bidirectional sound, enabling students, tutors and patients to interact directly with one another as they would during in-person bedside teaching. In the context of medical education, this technology enables clinicians to be able to deliver authentic bedside teaching in an immersive manner, providing maximal remote interaction with the patient and tutor. Remote teaching has gained a prominent role in medical education over recent years, becoming increasingly popular among students. ${ }^{6}$ It provides a convenient and accessible means of learning, which in turn influences student attendance. Attendance is vital to student learning, engagement levels and knowledge attainment. $^{7-9}$ At Imperial College London, we recognised 
that varying experiences on clinical attachments can bring discrepancies in the students' clinical exposure, such as seeing rare physical signs or uncommon pathology. We utilised the Microsoft HoloLens 2 to deliver parity through high-quality undergraduate teaching, bringing real patients to students' fingertips.

\section{Delivery}

A consultant in geriatric medicine, trained in the use of the Microsoft HoloLens 2, delivered this teaching on a general medical ward at a teaching hospital in London. The patient provided full consent before the session, understanding the purpose and nature of this teaching.

Our session was targeted at final-year medical students on their general medicine rotation who logged in remotely to view the live-streamed session via Microsoft Teams. This particular session focused on examination of the cardiovascular system of an 89-year-old inpatient. The consultant was able to ask the students how they would examine the patient, and then inspect the patient's surroundings and physical signs, identifying his Zimmer frame, a thoracotomy scar, venous graft scars and a raised jugular venous pressure. By being able to 'look through the consultant's eyes', they were able to observe the consultant's correct cardiovascular examination technique as if it was firsthand. Furthermore, there is the ability to play audio heart sounds during the examination for students to identify a cardiac murmur, further recreating what they would experience at the bedside.

\section{Innovation}

The more cutting-edge, novel element of the Microsoft HoloLens 2 is its ability to project holograms into the surrounding space and superimpose them on reality. Such holograms are viewable by both the consultant and the students and displayed beside the patient, thereby putting investigation findings or 3D anatomical models in the context of a real patient. During this session, holographic images of a chest X-ray displaying signs of heart failure, electrocardiography displaying atrial fibrillation and an infographic image of the $\mathrm{CHA}_{2} \mathrm{DS}_{2}$-VASc scoring system were projected onto a wall beside the patient (Fig 1). Students viewed the holograms and had the opportunity to view the appropriate findings from the images.

\section{Outcomes}

Thirty students attended this session. Students were able to communicate either by unmuting their microphone, or via the 'chat' provision on Teams. This flexibility afforded both confident and quiet students the opportunity to interact. The 35-minute session generated a total of 146 'chat' comments from 19 different students. There was also frequent direct communication between student, consultant and patient via the microphone. Though this bedside teaching was remotely delivered, students felt empowered to ask questions, engage in discussions with both the patient and tutor, and recognise clinical signs.

\section{Discussion}

With the educational landscape constantly evolving, being adaptable and creative with teaching is vital in training medical students. Teaching using novel technological equipment, such as the

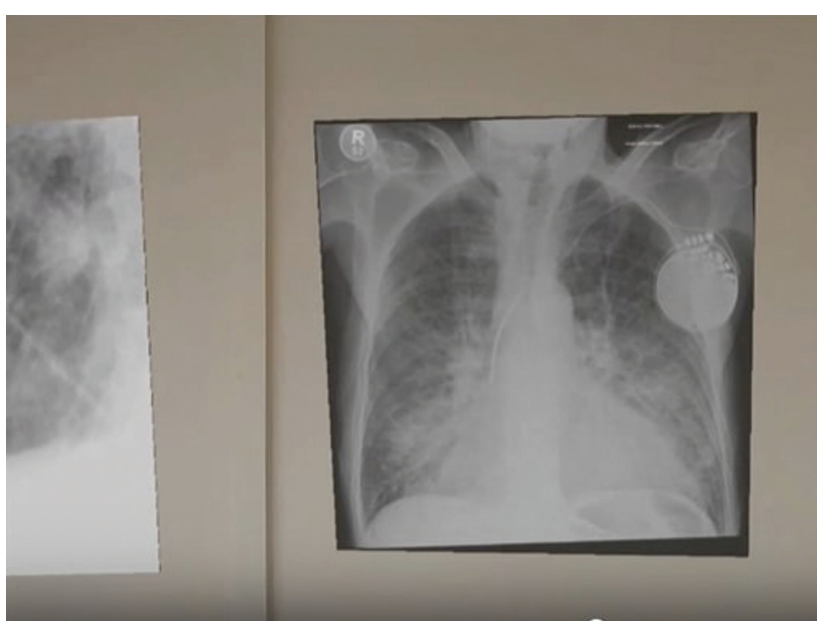

Fig 1. An example of an augmented reality chest X-ray. The image is virtually superimposed against a blank wall that the headset wearer is looking at, and the students can see through Teams. The holographic image can be manipulated by the headset wearer and moved around the room as they wish.

HoloLens 2, allows us to bring the unparalleled learning environment of the patient's bedside to students' fingertips in a unique and exciting way. As tutors and facilitators, we were fortunate to be part of a learning approach that, given today's climate, may become more prevalent and useful with each academic year.

Moreover, specifically with regards to general medicine and geriatrics, this session provided a valuable opportunity for students to gain exposure to frailty and multimorbidity, as well as consider the effects of heart failure in an older person. A study on medical student perceptions into geriatrics found that a majority expressed that they would like more teaching in this field. ${ }^{10}$ One barrier to bedside care of the elderly teaching may be that frail patients are unable to tolerate large numbers of medical students around them. However, this method allows them to be included in a comfortable environment, contemporaneously accessible to numerous students.

\section{Limitations and future direction}

An obvious limitation to this approach is that the students are not themselves examining the patient in person. However, our focus on interactivity intends to engage the students and still make it an active learning experience. Though more students are able to watch recordings of the session than would experience it in person, we are working on utilising the technology such that, in future, a larger proportion of the year group are able to watch the session in real time. This session was optional; more work is needed in obtaining data on engagement and knowledge attainment across a wider cohort.

\section{Conclusion}

Bedside teaching, first introduced by Sir William Osler, has for decades been the cornerstone of clinical teaching. Translating this concept remotely is possible through AR headsets such as the HoloLens 2, which can bring parity of bedside teaching to all students. Through the Teams HoloLens 2 sessions, we were able to deliver an inclusive and dynamic bedside teaching experience, with audience participation and empowerment of those less vocal. 
Following this session, we have been running fortnightly HoloLens 2 bedside teaching sessions with a particular focus on groups, such as with the elderly who may otherwise be under-represented. Such virtual experiences cannot replace the incomparable experience of in-person bedside teaching and will have their limitations. This form of teaching provides an adjunct to existing teaching methods and allows bedside teaching to continue even in challenging times. We hope that geriatrics, as well as other medical specialties, can benefit from this teaching tool and demonstrate the engaging and dynamic nature of clinical medicine to students.

Online teaching practices are an invaluable tool in andragogy, particularly as the world moves to a digital form of working, communicating and learning. Now, more than ever, it is important to innovate and develop proficiency in new digital learning tools and we hope that geriatrics and general medicine can play a leading role in this transformation.

\section{Acknowledgements}

We thank all the medical school teams at Imperial College School of Medicine involved in the organisation and delivery of this teaching session, and all students who participated. We also thank Health Education England for their support in delivering this teaching. Additionally, we particularly would like to thank Tom Yates and Thomas Hurkxkens for providing valuable support and laying the groundwork for this teaching session to be delivered.

\section{References}

1 Office for National Statistics. National population projections: 2018-based. ONS, 2019. www.ons.gov.uk/peoplepopulationandcommunity/populationandmigration/populationprojections/ bulletins/nationalpopulationprojections/2018based [Accessed 22 March 2021].

2 Meiboom AA, de Vries $\mathrm{H}$, Hertogh CM et al. Why medical students do not choose a career in geriatrics: a systematic review. BMC Med Educ. 2015;15:101.

3 Robbins TD, Crocker-Buque T, Forrester-Paton C et al. Geriatrics is rewarding but lacks earning potential and prestige: responses from the national medical student survey of attitudes to and perceptions of geriatric medicine. Age Ageing 2011;40;405-8.

4 Chróinín DN, Cronin E, Cullen W et al. Would you be a geriatrician? Student career preferences and attitudes to a career in geriatric medicine. Age Ageing 2013;42:654-7.

5 Bala L, Kinross ], Martin G et al. A remote access mixed reality teaching ward round. Clin Teach 2021;18:386-90.

6 O'Doherty D, Dromey M, Lougheed ] et al. Barriers and solutions to online learning in medical education - an integrative review. BMC Med Educ 2018;18:130.

7 McBrien JL, Cheng R, Jones P. Virtual spaces: Employing a synchronous online classroom to facilitate student engagement in online learning. The International Review of Research in Open and Distributed Learning 2009;10:1-17.

8 Kay D, Pasarica M. Using technology to increase student (and faculty satisfaction with) engagement in medical education. $A d v$ Physiol Educ 2019;43:408-13.

9 Tang B, Coret A, Qureshi A et al. Online lectures in undergraduate medical education: scoping review. JMIR Med Educ 2018;4:e11.

10 Curran M, Black M, Depp C et al. Perceived barriers and facilitators for an academic career in geriatrics: medical students' perspectives. Acad Psychiatry 2015;39:253-8.

Address for correspondence: Prof Amir H Sam, Imperial College London, Charing Cross Campus, Reynolds Building, St Dunstan's Road, London W6 8RP, UK.

Email:a.sam@imperial.ac.uk 